\title{
Systemic onset Juvenile Idiopathic Arthritis (SoJIA): a monocentric study of 114 patients
}

\author{
I Melki ${ }^{*}$, J Djadi-Prat ${ }^{2}$, AM Prieur ${ }^{1}$, C Job-Deslandre ${ }^{1}$, P Quartier ${ }^{1}$, C Elie ${ }^{2}$, B Bader Meunier ${ }^{1}$ \\ From 18th Pediatric Rheumatology European Society (PReS) Congress \\ Bruges, Belgium. 14-18 September 2011
}

\section{Background}

SoJIA is a rare paediatric disease, which may evolve in monophasic, polycyclic and chronic persistent course. Only a few studies have tried to bring out early factors of poor outcome that could lead to manage SoJIA according its severity at onset.

\section{Aim}

To describe presentation and outcome of SoJIA and to determine early clinical and laboratory characteristics associated to a poor outcome.

\section{Methods}

Retrospective study on SoJIA cases diagnosed by ILAR criteria between January 1985 and December 2005 in a tertiary paediatric rheumatology centre.

\section{Results}

During the study period, 114 children were included. Mean age at diagnosis was 4 years and 4 months (range, 4 months to 15.5 years). Familial history of autoimmunity was found in $27 \%$ of the patients. Glycosylated ferritin was low $(\leq 20 \%)$ for $79 \%$ of patients. Twelve percent of patients had macrophage activation syndrome, 2 patients anti-neutrophil cytoplasmic antibodies (ANCA) associated glomerulonephritis, 3 patients pulmonary restrictive syndrome without vertebral arthritis, and one patient Crohn's disease; $11 \%$ had positive antinuclear antibody (ANA) ( $\geq 1 / 160)$ at diagnosis. None developed neoplasia. Patients in whom diagnosis was made after 2000, and had been treated by biotherapy, especially anakinra, had less osteoarticular sequelae and lower inflammatory syndrome $(\mathrm{p}=0,004)$ than others (Figure 1). Early

\footnotetext{
* Correspondence: isabelle.melki@gmail.com

'Unité d'Immunologie, hématologie et Rhumatologie pédiatrique, Hôpital Necker Enfants malades, 75015 Paris, France

Full list of author information is available at the end of the article
}

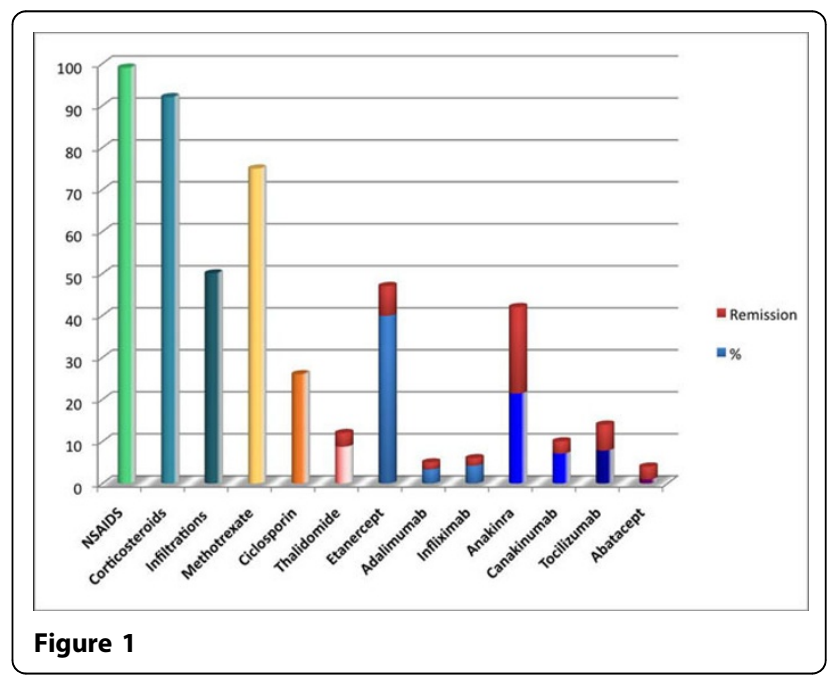

polyarticular and wrists involvements were associated to a poor outcome $(\mathrm{p}=0,009, \mathrm{p}=0,027)$.

\section{Conclusion}

This retrospective study suggests that 1) autoimmunity might be involved in the pathogenesis of SoJIA 2) early polyarticular and wrist involvements are associated with a poor outcome 3) anti-IL1 agents'use is associated with an improvement of the outcome.

\section{Author details}

'Unité d'Immunologie, hématologie et Rhumatologie pédiatrique, Hôpital Necker Enfants malades, 75015 Paris, France. ${ }^{2}$ Service de biostatistiques, Hôpital Necker Enfants malades, 75015 Paris, France.

Published: 14 September 2011

doi:10.1186/1546-0096-9-S1-P178

Cite this article as: Melki et al:: Systemic onset Juvenile Idiopathic

Arthritis (SoJIA): a monocentric study of 114 patients. Pediatric

Rheumatology 2011 9(Suppl 1):P178. 